\title{
TEMA -2018: El Proteosoma, sus Inhibidores y Toxicidad Cardíaca
}

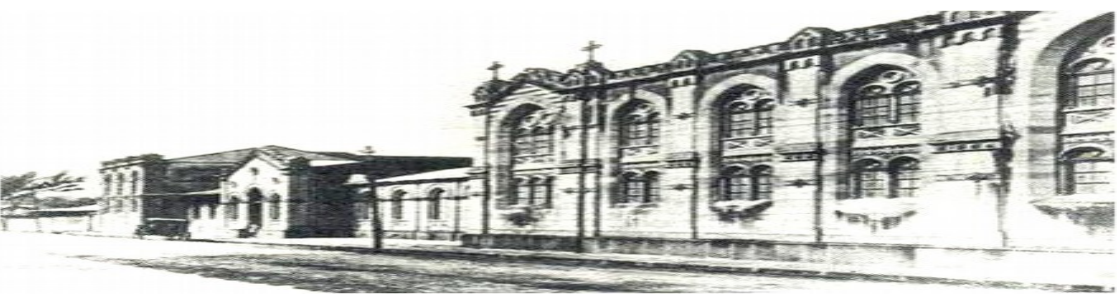

Haspital San quan de Dios. San José. Casta Rica. Fundada en 1845

Recibido:

$22 / 02 / 2018$

Aceptado:

Miguel A Rodriguez Pineda ${ }^{1}$

Max A. Méndez L ${ }^{2,3}$

${ }^{1}$ Departamento de Hematología Hospital México

2,3 Departamento de Hematología Hospital Calderón Guardia y Laboratorio de Oncología Experimental, Kantonsspital St. Gallen, Suiza. maxmendez@runbox.com

\section{RESUMEN}

El proteosoma es clave para mantenimiento de la homeostasis proteica. Los inhibidores del proteosoma son utilizados de forma rutinaria en un subgrupo de pacientes elegibles con mieloma multiple, sin embargo sus efectos terapéuticos de ven limitados por su toxicidad. Recientemente se ha extendido en entendimiento del Sistema Ubiquitina-Proteosoma en tumores sólidos y enfermedades neurológicas, razón por la cual el conocimiento y entendimiento de los efectos secundarios, en especial la cardiotoxicidad, son fundamentales para su posible expasión clínica.

\section{PALABRAS CLAVE}

Sistema Ubiquitina-Proteosoma. Autofagia. Inhibidor de Proteosoma. Cardiotoxicidad

\section{ABSTRACT}

The proteasome is key for proteostasis. Treatment with proteasome inhibitors in multiple myeloma patients is the hallmark of actual guide- lines, however, its therapeutic effect comes bound to toxicity. Recent advances in the understanding of the ubiquitin-proteasome system in solid tumors and neurological disorders preclude the expansion of the clinical use of the proteasome inhibitors out the haematological setting, therefore knowledge of the secondary effects, especially cardiotoxicity is essential.

\section{KEY WORDS}

Ubiquitin-Proteasome System. Autophagy. Proteasome Inhibitor. Cardiac Toxicity.

\section{INTRODUCCIÓN}

Los células cardíacas están expuestas a una gran cantidad de factores estresantes durante su período de vida y por ende son susceptibles de remodelado, cambios en el tamaño y pueden incluso inducir mecanismos metabólicos protectores. Esta actividad require de un enorme recambio proteico para mantener la estructura y función del sarcómero cardíaco íntegra, condición esencial para una célula sana y longeva ${ }^{1}$. Siendo el proteosoma y la homeostasis proteica una 
función celular fundamental, se ha invertido muchos recursos en el análisis funcional del proteosoma y la proteólisis en los cardiomiocitos. Dicha importancia se ve ejemplificada en las enfermedades donde algún elemento de la función sarcomérica es central en la fisiopatología, como es el caso de las miocardiopatías ${ }^{2,3}$ y en el daño por isquemia/reperfusion ${ }^{4}$.

Existen dos grandes mecanismos de degradación proteica, el Sistema Ubiquitina-Proteosoma (SUP) y la Autofagia. En general, se sabe que ambos sistemas tienen actividades que se superponen, pero su blanco de degradación son diferentes: el SUP es responsable de la degradación de proteínas funcionales (tipo I) e incorrectamente plegadas (tipo II), mientras que la autofagia degrada proteínas estructurales (tipo III) ${ }^{5}$.

El énfasis de esta revisión es es el uso de los inhibidores de proteosoma (IP) en el ámbito translacional, el mecanismo de funcionamiento del SUP y sus componentes, y principalmente la toxicidad cardíaca y la posibilidad de aprovechar su mecanismo de acción para modular su actividad su uso en situaciones que podría parecer contraproducente: el tratamiento de patologías cardíacas.

\section{DISCUSIÓN}

\section{El Sistema Ubiquitina-Proteosoma}

Los pasos críticos en el entendimiento del mecanismo y la función del proteosoma y el Sistema Ubiquitina-Proteosma (SUP) fueron establecidos por Ciechanover, Hershko y Rose, quienes recibieron el Premio Nobel en Química en el 2004 "por el descubrimiento de la degradación proteica mediada por ubiquitina" 6 . El SUP es responsable de la degradación de la mayoría de las proteínas en las células de mamíferos y su actividad se relaciona con la tasa de ubiquitinación, lo cual determina el balance entre la proporción de proteínas ubiquitinadas que son destruidas y la proporción de proteínas no ubiquitinadas que sobreviven ${ }^{7}$.
El SUP está conformado por el proteosoma, ubiquitina, la maquinaria de ubiquitinación y las enzimas deubiquitinasas ${ }^{4}$.

\section{Ubiquitina}

La ubiquitina, un polipéptido de 76 aminoácidos, es conjugada covalentemente con proteínas en los residuos de lisina en un proceso que require la acción secuencial de 3 enzimas. En breve, la enzima E1 (activadora de ubiquitina) se une a la ubiquitina en presencia de ATP para ser transferida posteriormente a una de las 40 enzimas E2 quienes a su vez, en conjunto con alguna de las cientos de ubiquitina-ligasas (E3), catalizan la transferencia de ubiquitina de E2 al sustrato ${ }^{8,9}$. Una vez finalizado el proceso, la cadena de poliubiquitina es removida por las enzimas Deubiquitinasas (Dub), es reciclada y preparada para un nuevo ciclo. (Fig 1).
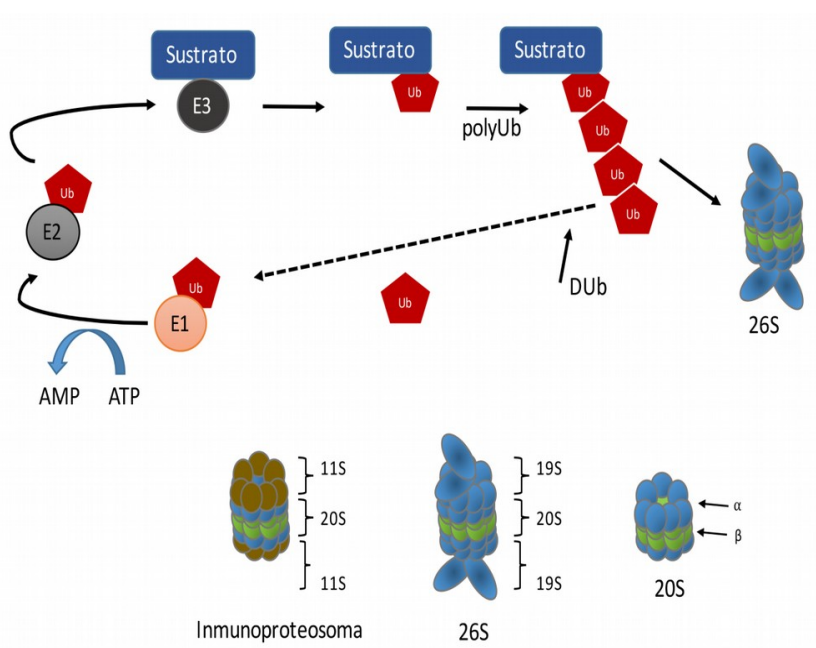

Figura 1. Esquema del Sistema UbiquitinaProteosoma

EI SUP está conformado por las enzimas ubiquitinligasas E1, E2, E3. La ubiquitina es cortada y reciclada por las Deubiquitinasas. Se muestra esquemáticamente la conformación del proteosoma $26 \mathrm{~S}$ y el inmunoproteosoma. Ub: Ubiquitina, Dub: Deubiquitinasas.

Fuente: Propia 


\section{Estructura y Localización del proteosoma $26 \mathrm{~S}$}

El proteosoma $26 \mathrm{~S}$ es un complejo multiproteico de 2.4MDa dependiente de ATP que se encuentra en el núcleo y citoplasma ${ }^{8}$. Consiste de una unidad catalítica central (20S) y dos subunidades regulatorias (19S) que catalizan en conjunto la degradación proteica ATP-dependiente por la vía ubiquitina (Fig 1).

Las proteínas son marcadas, reconocidas por el proteosoma $26 \mathrm{~S}$ y digeridas en péptidos cortos ${ }^{7}$.

\section{a. El proteosoma $20 \mathrm{~S}$}

Es la estructura proteolítica central y está formada por dos pares de anillos, cada uno de ellos con 7 subunidades.

La actividad constitutiva reside en los dos anillos internos que contienen las subunidades $\beta 1$ a $\beta 7$; de éstas, la $\beta 1$ posee actividad "caspase-like", la $\beta 2$ actividad "trypsin-like" y la $\beta 5$ actividad "chemotrypsin-like".

Algunas células, por ejemplo en retina y cerebro son capaces de intercambiar el proteosoma por el inmunoproteosoma, lo mismo sucede con algunas células tumorales como en el mieloma múltiple. En dichas estructuras, sus subunidades catalíticas se designan LMP2, MECL-1, and LMP7, sin embargo para efectos de simplicidad les designaremos $\beta 1 \mathrm{i}, \beta 2 \mathrm{i}$ y $\beta 5 \mathrm{i}$. Si lo resumimos de otra forma, los protesomas realizan cortes enzimáticos luego de residuos acídicos $(\beta 1)$, alcalinos $(\beta 2)$ e hidrofóbicos $(\beta 5)$.

\section{b. El complejo regulador $19 \mathrm{~S}$}

Es un complejo de 700kDa, 20 subunidades proteicas que se unen por ambos extremos al complejo 20S para formar el proteosoma 26S.
El complejo 11S se une a los extremos del proteosoma $20 \mathrm{~S}$ sin embargo, a diferencia del 19S no cataliza la degradación de proteínas grandes y juegan un papel importante en la producción de péptidos antigénicos para la presentación al Complejo Mayor de Histocompatibilidad Clase I.

SUP y Degradación proteica en el corazón

La degradación proteica en el corazón se basa es tres vías fundamentales que actúan de forma diferente pero complementaria: el SUP, la degradación por la vía autofágica/lisosomal y el Sistema Calpaína. En condiciones normales, el SUP a nivel cardíaco se ha vinculado con varias funciones vitales ${ }^{5,10-12}$ (Tabla 1), mientras la Autofagia y el Sistema Calpaína se han descrito con mayor profundidad recientemente.

1. El Sistema Ubiquitina-Proteosoma: en el corazón, el SUP es fundamental para mantener el recambio proteico y su función está asociada principalmente con la regulación de la atrofia cardíaca, inhibición de la hipertrofia y remodelado por estrés ${ }^{13,14}$. En el tejido cardíaco, las subunidades proteolíticas $\beta 1, \beta 2$ y $\beta 5$ son incorporadas en niveles mucho mayores que $\beta 1 \mathrm{i}, \beta 2 \mathrm{i}$ y $\beta 5 \mathrm{i}$, pero aún así la cantidad total de proteosoma es menor que que otros órganos. La especificidad del sustrato ocurre a nivel de las Ligasas de

Ubiquitina E3, las cuales se unen directamente a la proteína a ser degradada. Se han descrito 9 ligasas de ubiquitina, incluyendo el grupo de la familia MuRF (Muscle Ring Finger) que juegan un papel importante en la regulación de la estructura y función del músculo cardíaco y esquelético, y el grupo atrogin-1/MAFbx (Muscle Atrophy F-Box), las cuales son cardioprotectoras ${ }^{15,16}$

\section{c. El regulador $11 \mathrm{~S}$}


Tabla 1. Funciones del SUP en el cardiomiocito normal (Modificado de Ref 5)

\begin{tabular}{|l|l|}
\hline $\begin{array}{l}\text { Célula/Estructura } \\
\text { Diana }\end{array}$ & Función \\
\hline Canales de Potasio & Control de repolarización \\
\hline$\beta$-Adrenoceptores & $\begin{array}{l}\text { Regulación de degradación e } \\
\text { internalización del receptor } \\
\text { adrenérgico }\end{array}$ \\
\hline Conexina43 & Comunicación celular \\
\hline Canales de Sodio & $\begin{array}{l}\text { Regulación de excitabilidad y } \\
\text { conducción }\end{array}$ \\
\hline
\end{tabular}

Fuente: Modificado de $\operatorname{Ref}^{5}$

2. Autofagia en el Sarcómero Cardiaco: Autofagia es el proceso a través del cual proteínas, organelas y patógenos son removidos via degradación mediada por lisosomas (Fig.2). Existen dos grandes grupos: macroautofagia y microautofagia, para efectos de esta revisión nos referimos a macroautofagia, ya que es la más estudiada a nivel cardíaco. Los cardiomiocitos tienen vida prolongada y con poco potencial regenerativo, sin embargo, existe un nivel basal constante (pero bajo) de actividad autofágica en condiciones normales, lo cual permite una adecuada homeostasis celular ${ }^{17}$.

El proceso de autofagia inicia con la formación del autofagosoma, una estructura de doble membrana de origen reticular que se une al lisosoma, dentro del cual el contenido es degradado por hidrolasas. Los materiales reclutados son reciclados para producir estructuras de mayor complejidad (p.ej. proteínas y organellas).

Específicamente en el Sistema cardiovascular, la autofagia (o macroautofagia) tiene importancia a nivel al degradar el colágeno y favorecer el remodelado; en los cardiomiocitos al responder a isquemia, en el daño por reperfusion y remodelado inducido por sobrecarga por presión; en las células de músculo liso vascular al participar en inestabilidad de la placa aterosclerótica, y otras funciones que han demostrado que la autofagia ese un realidad un mecanismo de protección para el corazón, tales como actividades a nivel inflamatorio y en las células endoteliales ${ }^{18}$.

La Autofagia requiere una serie de proteínas conservadas evolutivamente llamadas Atg ${ }^{1}$, cuya importancia se ve ejemplificada en los modelos murinos. Los ratones adultos deficientes de Atg5 desarrollan desorganización sarcomérica, agregrados mitocondriales, hipertrofia cardiaca, dilatación ventricular y disfunción contráctil. Curiosamente, la deleción de Atg5 durante el desarrollo embrionario no ocasiona defectos funcionales, pero si produce insuficiencia cardíaca con incremento en las dimensiones ventriculares que se desarrolla a largo plazo con cambios estructurales meses antes de las manifestaciones clínicas ${ }^{19}$.

Igualmente, ratones sin expresión del gen Atg7 limitada a células endoteliales tienen un desarrollo capilar normal pero son incapaces de producir factor de von Willebrand (vWF), lo que sugiere que la autofagia es importante para el procesamiento y secreción de vWF pero no para el desarrollo vascular. Los cambios en la actividad autofágica son cuantificables por medio de microscopía, monitoreando la conversión de LC3-I a LC3-II y por medio de multiples proteínas asociadas a la autofagia, tanto por inmunohistoquímica como inmunofluorescencia ${ }^{18}$.

La autofagia tiene sus consecuencias tanto positivas como negativas, se sabe por ejemplo, que ésta aumenta luego del daño por isquemia/reperfusion, lo cual puede actuar 
como mecanismo protector al incrementar la cantidad de células viables al reducir la $\operatorname{apoptosis}^{20}$.

Igualmente, en cultivos celulares de cardiomiocitos atriales de ratón (HL-1), la apoptosis se incrementa al inhibir la autofagia lue-

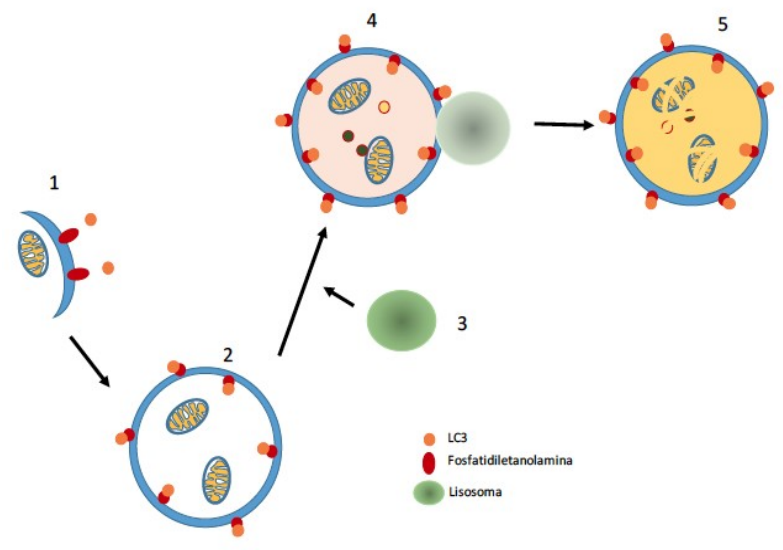

go de exponer las células al daño por isquemia-reperfusión. Lo que queda claro actualmente es que que uno de los principales desencadenantes de autofagia en el corazón es el acúmulo de agregados proteicos que genera incapacidad del SUP para deshacerse de las proteínas poli-ubiquitinadas ${ }^{21}$.

\section{Figura 2. Autofagia}

La (macro)autofagia amerita la formación de un complejo de iniciación que se expande formando un autofagosoma inmaduro, conocido también como "Estructura Preautofágica". 2) A medida que este crece, se encapsula y atrapa organellas en su interior, formando en autofagosoma. 3,4) La fusion con los lisosomas, provoca acidificación de sus componentes y liberación de hidrolasas que favorecen la degradación de sus contenidos, formando finalmente el autolisosoma (5). La proteína citoplasmática LC3 se encuentra en el ser humano como LC3A, B y C. LC3 $(A, B, C)-1$, de ubicación citosplasmática tiene un residuo de glicina C-terminal que es conjugado con fosfatildiletanolamina en LC3-II de membrana por medio de Atg5 y Atg7. Tanto LC3-I como LC3-II son marcadores de rutina para el estudio de autofagia. LC3: microtubule-associated protein light chain 3

Fuente: Propia

Tabla 2. Funciones de la Autofagia en el Sacó-

\begin{tabular}{|l|}
\hline Función \\
\hline Supresión de Apoptosis \\
\hline Remover Patógenos \\
\hline Remodelado celular \\
\hline Adaptación al estrés \\
\hline Reciclaje de nutrientes \\
\hline Recambio proteico \\
\hline Limitación del daño celular \\
\hline \multicolumn{1}{|c|}{ mero Cardíaco }
\end{tabular}

Fuente: Propia

3. El Sistema Calpaína: Las calpaínas son proteasas de serina calcio-dependientes. Las más estudiadas son calpaína-1 ( $\mu$-calpaína) y calpaína-2 (m-calpaína), nombradas según su respuesta a cantidades micro/milimolares de calcio. Ambas se encuentran en el corazón y se han relacionado con la degradación de proteínas miofibrilares tales como tropomiosina o troponinas. Se ha sugerido que las calpaínas son el Sistema degradativo responsable de la desestabilización de las miofibrillas que precede la proteólisis del sarcómero cardíaco 1. Esto surge de la hipótesis que la miofibrilla intacta debe primero ser desorganizada para que la proteólisis se pueda llevar a cabo, proceso que al parecer inicia marcando la troponina. Un dato interesante de notar es que la contribución 
del Sistema calpaína en la proteólisis pareciera ser mayor que vía del SUP ${ }^{22}$.

\section{Inhibidores de Proteosoma: Lecciones de los pacientes hematológicos.}

Estudios iniciales destinados a comprender el mecanismo de acción del SUP demostraron que el proteosoma es fundamental para el mantenimiento de la homeostasis proteica, incluyendo proteínas viculadas con el ciclo celular, proliferación celular y señalización pro- y anti-apoptótica.

Debido a su actividad central, por mucho tiempo se pensó que un medicamento que modificara su actividad manteniendo una dosis terapéutica sería difícil de desarrollar. Incluso, los IP no fueron diseñados como drogas de aplicación clínica, sino más bien, compuestos diseñados exclusivamente como una herramienta bioquímica/farmacológica para el estudio de las actividades proteolíticas del SUP ${ }^{23,24}$.

No obstante, inicialmente los resultados experimentales de líneas celulares demostraron que las células tumorales eran más sensibles a los inhibidores de proteosoma que las células normales ${ }^{25}$. Posteriormente, estudios preclínicos demostraron que las células humanas de mieloma múltiple eran más sensibles que las células mononucleares a la inhibición de crecimiento y apoptosis inducida por el inhibidor de proteosoma Bortezomib, abriendo el camino para la investigación clínica de los IP.

En el año 2000, el primer paciente con Mieloma Múltiple fue tratado con Bortezomib, alcanzando remisión completa luego de haber recibido previamente varias líneas de tratamiento fallido, con lo cual se inició una nueva era en el tratamiento del mieloma multiple ${ }^{26}$.

Tan solo 3 años después, se reportaba una sobrevida de 17.8 meses con una duración promedio de la respuesta de 14.3 meses para pacientes tratados con Bortezomib y ese mismo año era aprobado por la FDA como tratamiento para pacientes con mieloma multiple y recaída luego de dos líneas de tratamiento; para el año 2006, el Bortezomib era aprobado para su uso en pacientes con Linfoma de Células del Manto y en el 2008 como primera línea en mieloma multiple. Durante la última década, el resultado del Bortezomib ha sido tal que es considerado uno de los pilares del tratamiento de dicha patología hoy en día.

El mecanismo por el cual las células cancerosas, en especial las de mieloma multiple son más susceptibles que las células normales a los inhibidores de proteosoma está vinculado con la capacidad de los IP de inhibir la activación de NF- $k$ B por medio del TNF-alfa a través de la estabilización de $\mathrm{I} k \mathrm{~B}{ }^{27}$, bloqueando así las actividades proliferativas y antiapoptóticas de dicha vía e incrementando la susceptibilidad de las células tumorales a los agentes quimioterapéuticos. También se ha demostrado activación in vitro de caspasas y reducción in vivo de angiogenesis ${ }^{28}$. Sin embargo, como es esperable, dicho efecto terapéutico no es inocuo.

Los efectos secundarios varían desde trombocitopenia leve hasta fiebre y neutropenia con grados variables de neuropatía. Aún así, se ha logrado demostrar que la trombocitopenia es un efecto farmacocinético temporal, que no hay efectos en las células madre hematopoyéticas y se logró introducir cambios de dosis para el manejo de la neuropatía periférica ${ }^{9}$, con lo cual la cantidad de potenciales candidatos a dicha terapia se ha incrementado de forma significativa.

Curiosamente, no todos los inhibidores de proteosoma tienen el mismo perfil y éste parece estar directamente relacionado con su especificidad por una $u$ otra subunidad del proteosoma. El Bortezomib es un derivado boronato $\beta 1$ y $\beta 5$ específico reversible. El ixazomib, un análogo del Bortezomib administrado V.O, tiene una especificidad $\beta 5 \mathrm{y}$ el Carfilzomib, un derivado epoxiketona 
irreversible, es $\beta 5$ específico pero con efecto dual anti $\beta 5$ y $\beta 2$ a dosis altas, lo cual parece ser el mecanismo que explica la capacidad de dicho medicamento para producir apoptosis de células tumorales en adenocarcinoma de mama, efecto ausente con el Bortezomib.

Los efectos a nivel neuropático son menores con Carfilzomib que con Bortezomib, sin embargo se desconoce si es por un efecto específico de la droga o por la incapacidad de administrar dosis mayores con Carfilzomib debido a los efectos cardiovasculares.

\section{Mecanismo de Toxicidad Cardíaca}

Cuando los complejos 19S y 20S se asocian para formar el proteosoma 26S, las proteínas poliubiquitinadas son reconocidas, procesadas y deubiquitinadas antes de sufrir proteólisis. Es decir, si existe un incremento de proteínas poliubiquitinadas en el corazón se debe a una capacidad insuficiente del proteosoma 26S para degradar dichas proteínas. Como es evidente, tanto el incremento en la ubiquitinación como la disminución de la deubiquitinación son procesos igualmente patológicos, sin embargo en gran medida se desconoce cual ejerce un efecto mayoritario.

La mayoría de los inhibidores de proteosoma en práctica clínica son más efectivos contra la subunidad $\beta 5$, una característica que parece estar ligada con el desarrollo de fármacos mayoritariamente para su uso como terapia antitumoral ${ }^{29}$, sin embargo, dichos inhibidores podrían no ser apropiados para el corazón sano, debido a que han sido desarrollados con el objetivo de inducir apoptosis. De hecho, la evidencia demuestra que los inhibidores de proteosoma podrían ejercer un impacto negativo en el corazón tanto in vitro como in vivo al incrementar estrés oxidativo, disfunción mitocondrial y liberación de calcio del retículo endoplásmico ${ }^{30}$.

En la práctica clínica, la inhibición irreversible del proteosoma (p.ej Carfilzomib) se asocia a un síndrome común de insuficiencia cardíaca congestiva con disfunción sistólica y/o diastólica con disnea como síntoma principal ${ }^{31}$.

En dicho grupo de pacientes el BNP tiene un comportamiento predecible, al punto que podría ser utlizado como marcador para disfunción cardíaca en esta población; por otro lado, la troponina $\mathrm{T}$ parece no verse alterada y los daños estructurales son muy tempranos para ser detectados por ecocardiografía ${ }^{32}$. Si bien el BNP no parece correlacionarse con la gravedad de los síntomas es una herramienta útil como marcador subclínico de cardiotoxicidad por IPs.

La disfunción crónica primaria del proteosoma ocasiona alteración en la función y sobrevida del cardiomiocito, provocando un fenotipo de miocardiopatía restrictiva-hipertrófica, posiblemente relacionado con el incremento del estrés oxidativo $o$ estrés del retículo endoplásmico y la alteración en el balance de proteínas pro- y anti-apoptóticas ${ }^{33}$. La toxicidad resulta del acúmulo de proteínas anormales que forman oligómeros. El mecanismo del remodelado crónico mediado por la disminución de la actividad del proteosoma 26S continua sin ser entendido en su totalidad, sin embargo, existe evidencia que sugiere que la estimulación $\beta$ adrenérgica crónica conlleva a la desensibilización de la vía de señalización mediada por adenilato ciclasa y PKA en los cardiomiocitos ${ }^{29}$, algo que pareciera tener sentido si se considera que éste es, a su vez, una de las una de las bases farmacológicas del bloqueo $\beta$ adrenérgico en insuficiencia cardíaca. Finalmente, si se inhibe el proteosoma se incrementa 6 veces la conexin 43 ${ }^{12}$, la principal proteína a nivel cardíaco en las gap junctions encargada de permitir el paso de iones y moléculas pequeñas a lo largo del sincitio permitiendo la transmisión de señales.

Quedan muchos cuestionamientos por resolver: ¿todos los inhibidores de proteosoma son igualmente tóxicos para el cardiomiocito?, ¿existe algún método para modular su mecanismo de ac- 
ción y utilizarlo como una estrategia terapéutica en beneficio de los pacientes con patologías cardíacas? Lo que al menos en modelos murinos parece ser prometedor, y ¿cuál es el efecto de los IP en el cardiomiocito normal? No sabemos aún si nos encontramos ante un efecto de clase de droga, dosis dependiente, o tiempo dependiente o una combinación de varios.

Igualmente no se sabe con certeza si la toxicidad cardiac es por cardiotoxicidad directa, por daño endotelial o por inducción de apoptosis. Modelos murinos de constricción aórtica posterior a 3 semanas de tratamiento con IP, provocan un incremento de 2 a 3.5 veces en la actividad $\beta 5$ del proteosoma $26 \mathrm{~S}$ en los corazones con hipertrofia ${ }^{14}$, aunque parece ser una respuesta dependiente del nivel y del tiempo de la constricción, no del medicamento ${ }^{3}$. Se ha demostrado también una reducción en la hipertrofia cardíaca secundaria a sobrecarga por presión y la fracción de eyección del ventrículo izquierdo al utilizar IP en modelos murinos ${ }^{34}$. Es decir, se sugiere que el incremento de la actividad del proteosoma $26 \mathrm{~S}$ reduce el remodelado cardíaco y la insuficiencia cardíaca.

La explicación de la toxicidad de los IP pareciera ser un más compleja de lo esperable: el éxito de los IP en el tratamiento de la patologias cardíacas parece estar relacionado con la inhibición submáxima de la actividad del proteosoma ${ }^{29}$, ya que la inhibición persistente y severa produce insuficiencia cardíaca diastólica, en modelos murinos y en pacientes tratados crónicamente con el inhibidor irreversible de proteosoma Carfilzomib.

El infarto de miocardio es otro escenario en el cual el uso de los IP podría beneficiar a un subgrupo de pacientes. De acuerdo con Bulteau (2001) y Powell, la obstrucción in vivo de la arteria descendente anterior por al menos 30 minutos resulta en una disminución de la actividad proteasa ATP-dependiente. En modelos murinos de isquemia/reperfusion el uso de IP disminuyó la cantidad de leucocitos en los vasos coronarios y preservó la fracción de eyección ${ }^{35}$

\section{CONCLUSIONES}

Una situación que debe tomarse en consideración es que en humanos los síntomas se manifiestan en su mayoría cuando la insuficiencia cardíaca está establecida, al momento en que el daño estructural es irreversible y las actividades del proteosoma están casi ausentes, razón por la cual es lógico pensar que una estrategia apropiada sería el monitoreo frecuente la actividad proteosómica con el objetivo de detectar modificaciones sutiles que pudieran dictar cambios en la conducta terapéutica.

Los IP proteosoma han logrado mejorar la calidad de vida de los pacientes con mieloma multiple, una patología difícil de categorizar como "Enfermedad Terminal" debido a su cronicidad y su presentación en "picos". Si bien la toxicidad nivel cardíaco pareciera ser uno de los efectos secundarios menos frecuentes con los IP de primera generación, ésta se incrementa con la inhibición prolongada e irreversible del proteosoma, lo cual sugiere que el bloqueo submáximo del proteosoma o el monitoreo crónico de la función cardíaca indirectamente mediante niveles de BNP puede ser una estrategia factible para diagnosticar a tiempo el desarrollo de insuficiencia cardíaca congestiva.

No obstante útil, es una medida inespecífica y la ausencia de daño estructural cardíaco, la dificultad de la medición in vivo de la actividad del proteosoma, la dificultad (o imposibilidad) de la obtención de muestras cardíacas de humanos y el largo período de latencia desde el inicio de la terapia hasta la aparición de los síntomas complican el mantenimiento de una dosis anticancerígena efectiva con pocos efectos secundarios.

La toxicidad cardíaca no es esperable en los IP de primera generación, y el espectro de utilización de este grupo de medicamentos se ha extendido a cáncer de mama y neoplasias renales 
a nivel preclínico, lo que pronostica un incremento en su uso y por tanto la necesidad de comprender las bases biológicas y farmacológicas de dicha toxicidad.

En el campo oncológico, la perspectiva a mediano plazo se dirige a la investigación preclínica y el desarrollo de IP dirigidos específicamente a las subunidades del proteosoma sobreexpresadas por cada tipo de célula. También a nivel general el incremento en el entendimiento del SUPen pacientes con Alzheimer, Parkinson y Huntington preveen que haya un aumento en dicho tipo de medicamentos en el futuro cercano.

Por otro lado, existe la posibilidad de utilizar estos medicamentos como una estrategia alternativa para la terapia de ciertas patologías cardíacas, tales como el infarto de miocardio o la miocardiopatía hipertrófica al inducir apoptosis en los cardiomiocitos, tal y como ha sido demostrado en modelos animales.

\section{BIBLIOGRAFÍA}

1. Portbury AL, Willis MS, Patterson C. Tearin' Up My Heart: Proteolysis in the Cardiac Sarcomere. J Biol Chem. 2011;286(12):99299934. doi:10.1074/jbc.R110.170571.

2. Sanbe A, Osinska H, Saffitz JE, et al. Desmin-related cardiomyopathy in transgenic mice: A cardiac amyloidosis. Proc Natl Acad Sci United States Am . 2004;101(27):10132-10136. doi:10.1073/pnas.0401900101.

3. Tsukamoto O, Minamino T, Okada K, et al. Depression of proteasome activities during the progression of cardiac dysfunction in pressure-overloaded heart of mice. Biochem Biophys Res Commun. 2006;340(4):1125-1133. doi:https://doi.org/10.1016/j.bbrc.2005.12.120.

4. Calise J, Powell SR. The ubiquitin proteasome system and myocardial ischemia. Am $\mathrm{J}$ Physiol Heart Circ Physiol. 2013;304(3):H33749. doi:10.1152/ajpheart.00604.2012.
5. Barac YD, Emrich F, Krutzwakd-Josefson E, et al. The ubiquitin-proteasome system: A potential therapeutic target for heart failure. $\mathrm{J}$ Hear Lung Transplant. 2017;36(7):708-714. doi:10.1016/j.healun.2017.02.012.

6. Nobel Prize Foundation. The Nobel Prize in Chemistry 2004. https://www.nobelprize.org/nobel_prizes/chemistry/laureates/2004/. Published 2004. Accessed August 29, 2017.

7. Collins GA, Goldberg AL. The Logic of the 26S Proteasome. Cell. 2017;169(5):792-806. doi:10.1016/j.cell.2017.04.023.

8. Adams J. The proteasome: a suitable antineoplastic target. Nat Rev Cancer. 2004;4(5):349-360.

http://dx.doi.org/10.1038/nrc1361.

9. Esseltine D-L, Mulligan G. An Historic Perspective of Proteasome Inhibition. Semin Hematol. 2012;49(3):196-206. doi:http://dx.doi.org/10.1053/j.seminhematol.2012.04.009.

10. Shenoy SK, McDonald PH, Kohout TA, Lefkowitz RJ. Regulation of receptor fate by ubiquitination of activated beta 2-adrenergic receptor and beta-arrestin. Science. 2001;294(5545):1307-1313.

doi:10.1126/science.1063866.

11. van Bemmelen MX, Rougier J-S, Gavillet $\mathrm{B}$, et al. Cardiac voltage-gated sodium channel Nav1.5 is regulated by Nedd4-2 mediated ubiquitination. Circ Res. 2004;95(3):284291. doi:10.1161/01.RES.0000136816.05109.89.

12. Laing JG, Beyer EC. The Gap Junction Protein Connexin43 Is Degraded via the Ubiquitin Proteasome Pathway. J Biol Chem. 1995;270(44):26399-26403.

doi:10.1074/jbc.270.44.26399.

13. Glass DJ. Signalling pathways that mediate skeletal muscle hypertrophy and atrophy. Nat Cell Biol. 2003;5(2):87-90. http://dx.doi.org/10.1038/ncb0203-87.

14. Depre C, Wang Q, Yan L, et al. Activation of the Cardiac Proteasome During Pressure Overload Promotes Ventricular Hypertrophy. 
Circulation. 2006;114(17):1821 LP-1828. http:/circ.ahajournals.org/content/114/17/1821.a bstract.

15. Spencer JA, Eliazer S, Ilaria RL, Richardson JA, Olson EN. Regulation of Microtubule Dynamics and Myogenic Differentiation by Murf, a Striated Muscle Ring-Finger Protein. J Cell Biol. 2000;150(4):771 LP-784. http://jcb.rupress.org/content/150/4/771.abstract.

16. Gomes MD, Lecker SH, Jagoe RT, Navon A, Goldberg AL. Atrogin-1, a muscle-specific F-box protein highly expressed during muscle atrophy. Proc Natl Acad Sci. 2001;98(25):14440-14445.

doi:10.1073/pnas.251541198.

17. Ma S, Wang Y, Chen Y, Cao F. The role of the autophagy in myocardial ischemia/reperfusion injury. Biochim Biophys Acta - Mol Basis Dis. 2015;1852(2):271-276. doi:http://dx.doi.org/10.1016/j.bbadis.2014.05.01 0 .

18. Lavandero S, Chiong M, Rothermel BA, Hill JA. Autophagy in cardiovascular biology. J Clin Invest. 2015;125(1):55-64. doi:10.1172/JCI73943.

19. Nakai A, Yamaguchi O, Takeda T, et al. The role of autophagy in cardiomyocytes in the basal state and in response to hemodynamic stress. Nat Med. 2007;13(5):619-624. http://dx.doi.org/10.1038/nm1574.

20. Yan L, Vatner DE, Kim S-J, et al. $A u$ tophagy in chronically ischemic myocardium. Proc Natl Acad Sci United States Am . 2005;102(39):13807-13812.

doi:10.1073/pnas.0506843102.

21. Tannous $\mathrm{P}, \mathrm{Zhu} \mathrm{H}$, Nemchenko A, et al. Intracellular Protein Aggregation Is a Proximal Trigger of Cardiomyocyte Autophagy. Circulation. 2008;117(24):3070 LP-3078. http://circ.ahajournals.org/content/117/24/3070.abstract.

22. Galvez AS, Diwan A, Odley AM, et al. Cardiomyocyte Degeneration With Calpain Deficiency Reveals a Critical Role in Protein Homeostasis. Circ Res. 2007;100(7):1071 LP-1078. http://circres.ahajournals.org/content/100/7/1071. abstract.

23. Orlowski RZ, Kuhn DJ. Proteasome Inhibitors in Cancer Therapy: Lessons from the First Decade. Clin Cancer Res. 2008;14(6):1649 LP-1657.

http://clincancerres.aacrjournals.org/content/14/6 1649.abstract.

24. Vinitsky A, Michaud C, Powers JC, Orlowski M. Inhibition of the chymotrypsin-like activity of the pituitary multicatalytic proteinase complex. Biochemistry. 1992;31(39):9421-9428.

25. SHINOHARA K, TOMIOKA M, NAKANO H, TONÉ S, ITO H, KAWASHIMA S. Apoptosis induction resulting from proteasome inhibition. Biochem J. 1996;317(2):385 LP-388.

http://www.biochemj.org/content/317/2/385.abstract.

26. Orlowski RZ, Stinchcombe TE, Mitchell BS, et al. Phase I Trial of the Proteasome Inhibitor PS-341 in Patients With Refractory Hematologic Malignancies. J Clin Oncol. 2002;20(22):4420-4427.

doi:10.1200/JCO.2002.01.133.

27. Orlowski RZ, Baldwin Jr AS. $N F_{k} B$ as a therapeutic target in cancer. Trends Mol Med. 2017;8(8):385-389. doi:10.1016/S14714914(02)02375-4.

28. LeBlanc R, Catley LP, Hideshima T, et al. Proteasome Inhibitor PS-341 Inhibits Human Myeloma Cell Growth \& and Prolongs Survival in a Murine Model. Cancer Res. 2002;62(17):4996 LP-5000. http://cancerres.aacrjournals.org/content/62/17/4996.abstract.

29. Drews O, Taegtmeyer H. Targeting the ubiquitin-proteasome system in heart disease: the basis for new therapeutic strategies. Antioxid Redox Signal. 2014;21(17):2322-2343. doi:10.1089/ars.2013.5823.

30. Paniagua Soriano G, De Bruin G, Overkleeft HS, Florea BI. Toward understanding induction of oxidative stress and apoptosis by proteasome inhibitors. Antioxid Redox Signal. 2014;21(17):2419-2443.

doi:10.1089/ars.2013.5794. 
31. Grandin EW, Ky B, Cornell RF, Carver J, Lenihan DJ. Patterns of cardiac toxicity associated with irreversible proteasome inhibition in the treatment of multiple myeloma. J Card Fail. 2015;21(2):138-144.

doi:10.1016/j.cardfail.2014.11.008.

32. Koulaouzidis G, Lyon AR. Proteasome Inhibitors as a Potential Cause of Heart Failure. Heart Fail Clin. 2017;13(2):289-295. doi:10.1016/j.hfc.2016.12.001.

33. Herrmann J, Wohlert C, Saguner AM, et al. Primary proteasome inhibition results in cardiac dysfunction. Eur J Heart Fail. 2013;15(6):614-623. doi:10.1093/eurjhf/hft034.

34. Hedhli N, Lizano P, Hong C, et al. Proteasome inhibition decreases cardiac remodeling after initiation of pressure overload. Am J Physiol - Hear Circ Physiol. 2008;295(4):H1385 LPH1393.

http://ajpheart.physiology.org/content/295/4/H13 85.abstract.

35. Campbell B, Adams J, Shin YK, Lefer AM. Cardioprotective Effects of a Novel Proteasome Inhibitor Following Ischemia and Reperfusion in the Isolated Perfused Rat Heart. J Mol Cell Cardiol. 2017;31(2):467-476. doi:10.1006/jmcc.1998.0880.

\section{CONFLICTO DE INTERÉS Y/O AGRADECIMIENTOS}

Los autores declaran que no existió ningún conflicto de interés en el presente reporte. 